\title{
TEVAR for Acute Symptomatic DeBakey Tip III Dissection
}

\section{๑) Onder Ozturk1, ๑ Utkan Sevuk2}

${ }^{1}$ University of Health Sciences, Diyarbakır Gazi Yasargil Education and Research Hospital, Clinic of Cardiology, Diyarbakır, Turkey

2University of Health Sciences, Diyarbakır Gazi Yasargil Education and Research Hospital, Clinic of Cardiovascular Surgery, Diyarbakır, Turkey

\begin{abstract}
Aortic dissection is a challenging life-threatening vascular emergency. Thoracic endovascular aortic repair (TEVAR) represents a minimally invasive technique alternative to conventional open surgical reconstruction for the treatment of thoracic aortic pathologies. In this report, we presented a
\end{abstract}

\section{Introduction}

Aortic dissection (AD) occurs when the blood flows between the layers of the wall of the aorta. It can rapidly lead to death due to insufficient coronary perfusion or instantaneous rupture of the aorta, which is a severe and fatal complication requiring emergent management. AD is a challenging life-threatening vascular emergency. DeBakey type I or II (Stanford type A) AD involving the ascending aorta is treated by urgent surgical intervention, while DeBakey type III (Stanford type B) AD involving the descending thoracic aorta (DeBakey type IIIa) or thoracoabdominal aorta (DeBakey type IIIb) is managed 92-year-old female patient complicated with DeBakey Tip III aortic dissection successfully treated with TEVAR.

Keywords: TEVAR, aortic dissection, percutaneous therapy

medically or by surgical or endovascular intervention when it is complicated ${ }^{(1)}$. Thoracic endovascular aortic repair (TEVAR) represents a minimally invasive technique alternative to conventional open surgical reconstruction for the treatment of thoracic aortic pathologies. Rapid advances in endovascular technology and procedural breakthroughs have contributed to a dramatic transformation of the entire field of thoracic aortic surgery ${ }^{(2)}$. EVAR procedures can be challenging and, at times, extraordinarily difficult. They require seasoned endovascular experience and refined skills $^{(3)}$. Of all endovascular procedures, meticulous assessment of anatomy and preoperative procedure

Address for Correspondence: Onder Ozturk, University of Health Sciences, Diyarbakır Gazi Yasargil Training and Research Hospital, Clinic of Cardiology, Diyarbakır, Turkey

Phone: +90 5332652602 e-mail: droozturk21@hotmail.com ORCID: orcid.org/0000-0003-4204-0599

Received: 12.06.2019 Accepted: 06.02.2020

Cite this article as: Ozturk O, Sevuk U. TEVAR for Acute Symptomatic DeBakey Tip III Dissection. EJCM 2020;8(1):56-58.

DOI: 10.32596/ejcm.galenos.2020.06.033

${ }^{\circ}$ Copyright 2020 by Heart and Health Foundation of Turkey (TÜSAV) / E Journal of Cardiovascular Medicine published by Galenos Publishing House. 
planning are absolutely paramount to produce optimal outcomes. In this report, we presented a 92-year-old female patient complicated with DeBakey Tip III aortic dissection successfully treated with TEVAR.

\section{Case Report}

A 92-year-old female patient was admitted to our hospital due to complaints of acute-onset chest and back pain for 10 hours. Patient had had hypertension for 20 years with an uncontrolled high blood pressure found on admission. The blood pressure was measured as 185/70 $\mathrm{mm} \mathrm{Hg}$ in the right arm, and $130 / 65 \mathrm{~mm} \mathrm{Hg}$ in the left arm with a pulse rate of 106 beats/minute. Computed tomography angiography revealed a dissection flap in the descending aorta beginning after the left subclavian artery with an ending level at the common iliac arteries bifurcation. Other vital visceral arteries were not affected, as illustrated in Figure 1. The patient was hospitalized. She

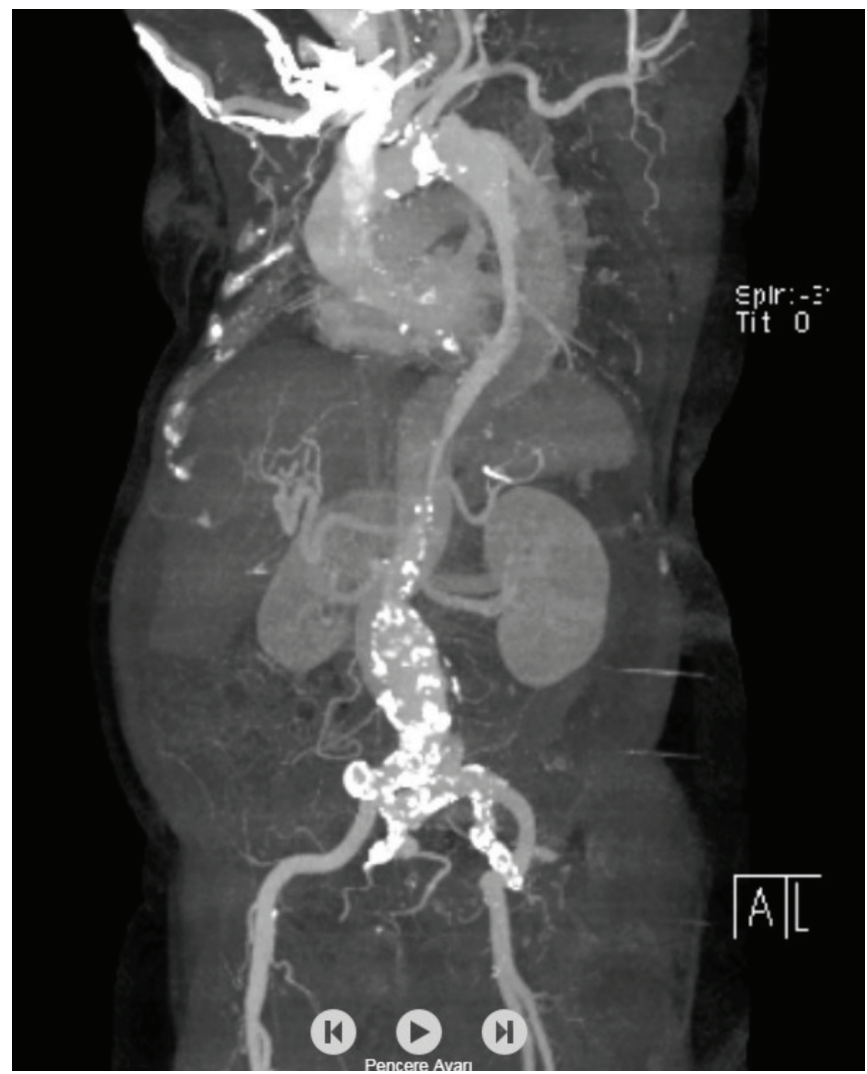

Figure 1. CT Angiography of DeBakey Tip III aortic dissection CT: Computed tomography had advanced age. Therefore, open surgery was highly risky for morbidity and mortality. Therefore, the patient underwent TEVAR. The vascular stents were selected based upon the measurement of the vascular diameter (Figure 2). 38x16 mm graft stent covered the origin of the left subclavian artery (Figure 3). No stent endoleak or dislocation occurred. The patient remained stable after TEVAR. The patient was discharged the next day after TEVAR.

\section{Discussion}

Since the introduction of aortic stent grafting for the treatment of thoracic aortic aneurysms, TEVAR has gained widespread clinical application as a less invasive technique for DeBakey type III aortic dissection compared to the open surgery ${ }^{(4)}$. Our case had advanced age. Therefore, open surgery was highly risky for morbidity and mortality. However, the use of TEVAR is associated with the risk of postoperative complications. and even high rate of morbidity and mortality. Indications for TEVAR in treating thoracic aortic aneurysms include paraplegia, visceral ischemia, acute rupture, chronic aneurysm, etc.
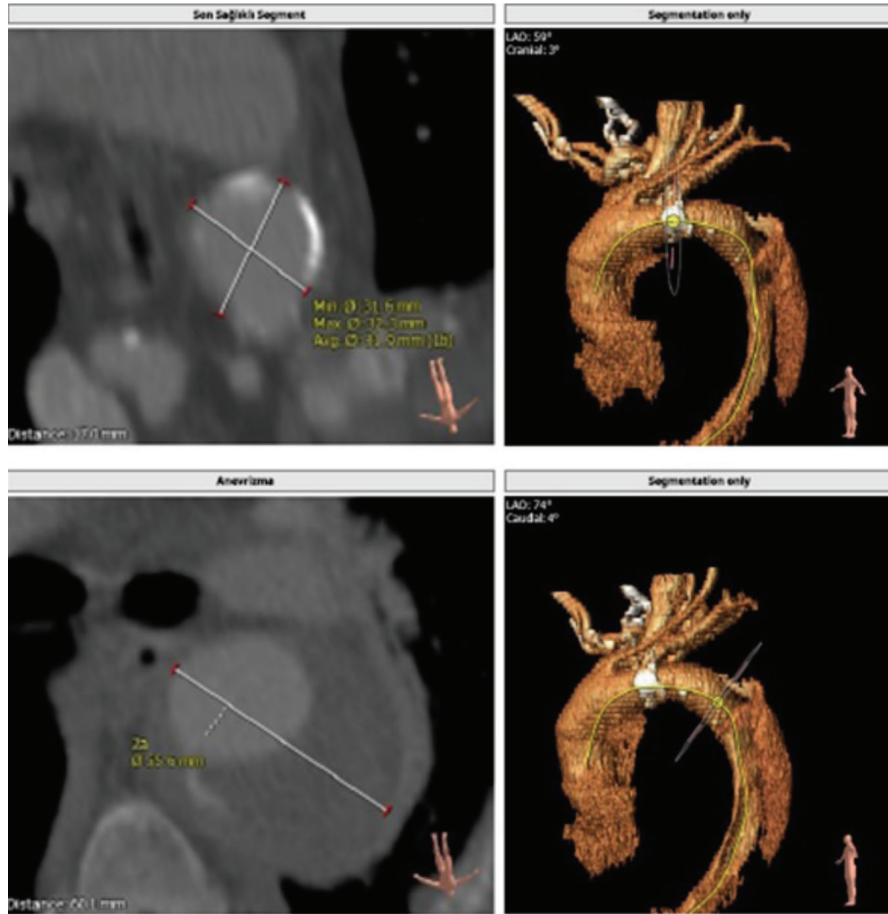

Figure 2. Calculation of graft stent sizing 


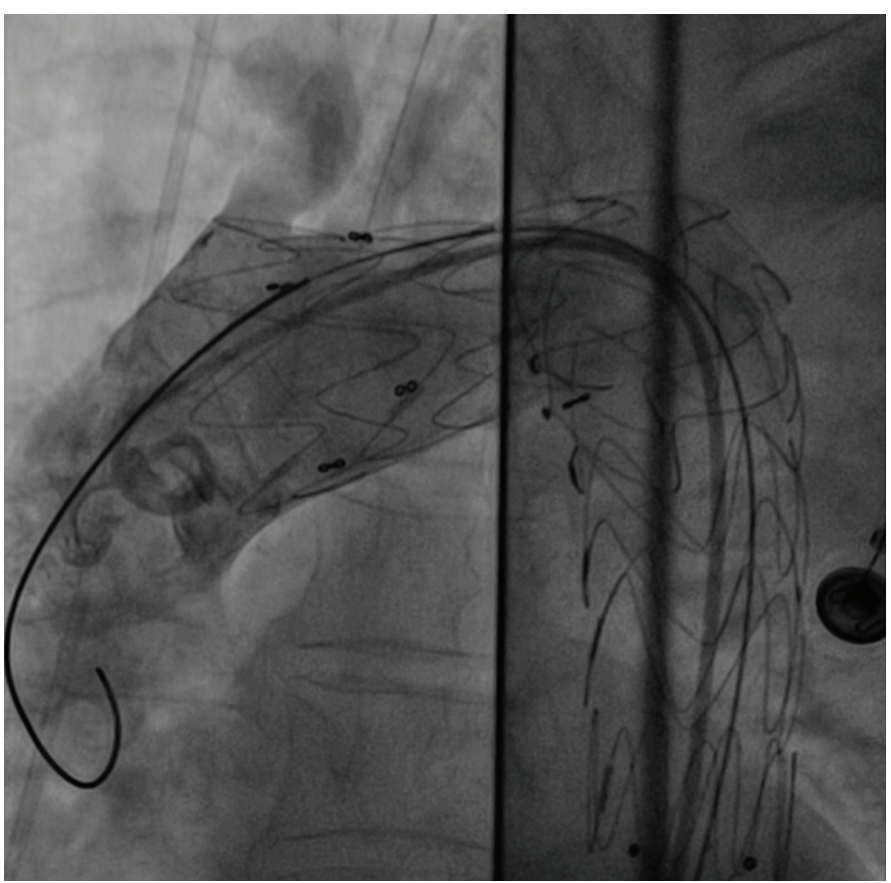

Figure 3. Graft stenting implantation

Paraplegia is the most severe acute complication after TEVAR. Previous studies have demonstrated that the incidence of both immediate and delayed paraplegia in patients undergoing TEVAR can be as high as $12 \%$, compared to $2 \%$ to $21 \%$ in their counterparts after open surgery ${ }^{(5,6)}$.

Paraplegia is due to spinal cord injury (SCI). The simultaneous closure of two independent arterial spinal cord vessels, and intraoperative hypotension, has been shown to be the most important risk factor for symptomatic $\mathrm{SCI}^{(5)}$. It has also been suggested with regard to the optimal size of the stent graft that a device with the same diameter as the true lumen diameter, or with a $10 \%$ increase beyond this diameter. Available stent graft diameters are 20-46 $\mathrm{mm}$. In this case, a dissection flap in the descending aorta beginning after the left subclavian artery with an ending level at the common iliac arteries bifurcation. Dissection flap was very long in this patient. Coverage of all of the dissected segment with graft stenting is increasing the risk of SCI. Therefore, we used only one graft stent for the prevention of paraplegia. Patient was treated successfully.

\section{Ethics}

Informed Consent: Informed consent was obtained from the patients.

Peer-review: Externally and internally peer-reviewed.

\section{Authorship Contributions}

Surgical and Medical Practices: O.O., U.S., Concept: O.O., U.S., Design: O.O., U.S., Data Collection or Processing: O.O., U.S., Analysis or Interpretation: O.O., U.S., Literature Search: O.O., U.S., Writing: O.O., U.S.

Conflict of Interest: No conflict of interest was declared by the authors.

Financial Disclosure: The authors declared that this study received no financial support.

\section{References}

1. Erbel R, Aboyans V, Boileau C, et al. 2014 ESC Guidelines on the diagnosis and treatment of aortic diseases: Document covering acute and chronic aortic diseases of the thoracic and abdominal aorta of the adult. The Task Force for the Diagnosis and Treatment of Aortic Diseases of the European Society of Cardiology (ESC). Eur Heart J 2014 Nov 1;35:2873-926.

2. Steuer J, Eriksson MO, Nyman R, et al. Early and long-term outcome after thoracic endovascular aortic repair (TEVAR) for acute complicated type $\mathrm{B}$ aortic dissection. Eur J Vasc Endovasc Surg 2011;41:318-23.

3. Patel SR, Allen C, Grima MJ, et al. A Systematic Review of Predictors of Reintervention After EVAR: Guidance for Risk-Stratified Surveillance. Vasc Endovascular Surg 2017;51:417-28.

4. Krol E, Panneton JM. Uncomplicated acute type B aortic dissection: selection guidelines for TEVAR. Ann Vasc Dis 2017;10:165-9.

5. Sueda T, Takahashi S. Spinal cord injury as a complication of thoracic endovascular aneurysm repair. Surg Today. 2018;48:473-7.

6. Patterson BO, Cobb RJ, Karthikesalingam A, et al. A systematic review of aortic remodeling after endovascular repair of type B aortic dissection: methods and outcomes. Ann Thorac Surg 2014;97:588-95. 